\title{
Consideration of therapeutic approach to advanced colorectal cancer in elderly patients
}

\author{
Yasuhiro Inoue, Yuji Toiyama, Koji Tanaka, Yasuhiko Mohri, Masato Kusunoki \\ Department of Gastrointestinal and Pediatric Surgery, Division of Reparative Medicine, Institute of Life Sciences, Mie University \\ Graduate School of Medicine, Tsu, Mie, Japan.
}

Received November 29, 2013; Revised December 23, 2013; Accepted December 27, 2013; Published Online January 03, 2014

\section{Scientific $\mathcal{N}$ ote}

\begin{abstract}
Colorectal cancer (CRC) is predominantly a disease of elderly and is a major cause of morbidity and mortality in the elderly population. The increased availability of treatment options for CRC has made it more difficult for clinicians to decide on the optimal therapeutic approach in elderly patients, because of the potential for poorer outcomes due to an increased burden of comorbidities, functional dependency, and limited life expectancy. It is necessary to determine which elderly patients are likely to benefit from active cancer therapy, and the establishment of treatment markers for multimodality approaches is eagerly awaited. Elderly cancer patients are at risk of exposure to various intrinsic inflammatory mediators, such as tumor-generating cytokines and surgery-induced pro-inflammatory cytokines. It is therefore important to understand the immunological changes occurring in the elderly and to adjust treatment strategies accordingly to reduce the morbidity and mortality associated with multimodality therapy for CRC that induce systemic inflammation. Several inflammation-based factors such as the Glasgow Prognostic Score (GPS) may reflect the balance between tumor progression and host-related immunity, especially in elderly CRC patients. Appropriate selection criteria for multimodality therapy in elderly CRC patients may include not only tumor characteristics, but also host- and/or treatment-related factors such as comorbidities or surrogate markers using inflammation-based factors.
\end{abstract}

Keywords: Colorectal cancer; Multimodality therapy; Systemic inflammatory response; Glasgow Prognostic Score

\section{Introduction}

The elderly population is increasing rapidly in developed countries, and cancer remains a major public health problem affecting more elderly than younger individuals. Clinicians need to evaluate elderly cancer patients carefully, because of the increased likelihood of comorbidities and age-specific organ-function deterioration, both of which have implications for cancer therapy. Colorectal cancer (CRC) is the commonest malignancy worldwide, and is largely a disease of the elderly. Various treatment modalities have been used to prolong survival in patients with CRC, including surgery, radiotherapy and chemotherapy, and this multimodal thera-

Corresponding author: Yasuhiro Inoue, MD; Department of Gastrointestinal and Pediatric Surgery, Division of Reparative Medicine, Institute of Life Sciences, Mie University Graduate School of Medicine, Edobashi 2-174, Tsu 514-8507, Japan.

Email:yasinoue@clin.medic.mie-u.ac.jp

Cite this article as:

Inoue Y, Toiyama Y, Tanaka K, Mohri Y, Kusunoki M. Consideration of therapeutic approach to advanced colorectal cancer in elderly patients. Int J Cancer Ther Oncol 2014; 2(1):02014.

DOI: $10.14319 / \mathrm{ijcto.0201.4}$ peutic approach to CRC represents a good role model for the medical and social optimization of cancer treatments in elderly patients.

Surgery is the mainstay of CRC treatment; however, modern chemotherapy with molecular-targeted agents has increased the survival time to more than 2 years, even in patients with inoperable metastases. Recent multimodal approaches involving chemotherapy, radiotherapy and surgery for primary and/or metastatic lesions, alone or in combination, have been proposed to prolong the survival of patients with advanced CRC even further. However, the increased availability of treatment options has made it more difficult for clinicians to decide on the optimal therapeutic approach, especially in elderly patients.

The elderly CRC population is a heterogeneous group, including patients with an otherwise excellent health status, as well as those with comorbid conditions, functional dependency, and limited life expectancy. Elderly patients are therefore sometimes undertreated compared with younger patients, because of clinician preferences. Published results for 
surgical morbidity and mortality, and for the safety of chemotherapy in elderly patients with CRC are also conflicting, ${ }^{1,2}$ and scientific evidence for the efficacy of modern multimodality therapy in advanced CRC is scarce. However, we recently published the results of a study involving the treatment of elderly patients with multimodality therapy for metastatic or recurrent CRC. ${ }^{3}$

Some of the elderly patients derived as much benefit from aggressive multimodality therapy as younger patients. The prognostic factors for elderly patients were host- and treatment-related, whereas the prognostic factors for younger patients were tumor-related. This evidence suggests that therapeutic decisions should be made on an individual basis, taking into account the patient's overall clinical status and preferences, and should not be based merely on chronological age. It is therefore necessary to determine which elderly patients are likely to benefit from active cancer therapy, and the establishment of treatment markers for multimodality approaches is eagerly awaited.

There is also clear evidence to indicate that elderly patients are more susceptible to postoperative infections than younger patients, which may be related to the pro-inflammatory status of elderly patients. ${ }^{1}$ Elderly patients with cancer are also at risk of exposure to various intrinsic inflammatory mediators, such as tumor-generating cytokines and surgery-induced pro-inflammatory cytokines. It is therefore important to understand the immunological changes occurring in the elderly and to adjust treatment strategies accordingly to reduce the morbidity and mortality associated with cancer therapies that induce systemic inflammation.

Increasing evidence that host or immune responses are important prognostic indicators has been associated with the development of a variety of prognostic scores based on the presence of the systemic inflammatory response. The Glasgow Prognostic Score (GPS), an inflammation-based score that includes serum C-reactive protein (CRP) and albumin levels, is currently one of the most useful prognostic indicators in patients with advanced cancer. ${ }^{4}$ Although the reasons why elevated CRP correlates with poor long-term outcome in cancer patients is not fully understood, several pro-inflammatory cytokines expressed in the tumor environment, such as interleukin-1(IL-1), IL-6, and tumor necrosis factor- $\alpha$, may affect the malignant potential of tumor cells. ${ }^{5}$ IL-6 is known to stimulate the acute-phase expression of CRP. We previously reported that metabolic compliance against intrinsic IL-6 may be compensated for in the stable preoperative period, but may deteriorate as a result of surgical stress in elderly CRC patients with malnutrition. ${ }^{6}$

These results also suggest that the change in systemic inflammatory response during cancer therapy might reflect compromised immunity in elderly patients with CRC. Indeed, we detected a change in modified GPS after chemo- therapy in patients with CRC, suggesting that modified GPS may thus be a potential prognostic marker in elderly patients receiving multimodality therapy.3,7 GPS is simply a marker of inflammation, and the cause-effect relationship between a systemic inflammatory response and cancer survival is also uncertain; however, the combination of several inflammation-based factors might reflect the balance between tumor progression and host-related immunity, especially in elderly cancer patients.

In conclusion, chronological age alone should not be a contraindication to multimodality cancer therapy in elderly patients with CRC, and the use of active multimodality therapy may be a valuable approach for improving survival in these patients. Furthermore, appropriate selection criteria for multimodality therapy in elderly CRC patients may include not only tumor characteristics, but also host- and/or treatment-related factors such as comorbidities or surrogate markers such as GPS.

\section{Conflict of interest}

The authors declare that they have no conflicts of interest. The authors alone are responsible for the content and writing of the article.

\section{References}

1. Colorectal Cancer Collaborative Group. Surgery for colorectal cancer in elderly patients: a systematic review. Lancet 2000; 356:968-74.

2. Serra-Rexach JA, Jimenez AB, García-Alhambra $\mathrm{MA}$, et al. Differences in the therapeutic approach to colorectal cancer in young and elderly patients. Oncologist 2012; 17:1277-85.

3. Inoue Y, Toiyama Y, Tanaka K, Mohri Y, Kusunoki M. Outcome of multimodality therapy for elderly colorectal cancer patients. Cancer and Clinical Oncology 2013; 2:70-80.

4. Proctor MJ, Morrison DS, Talwar D, et al. An inflammation-based prognostic score (mGPS) predicts cancer survival independent of tumour site: a Glasgow Inflammation Outcome Study. $\mathrm{Br} J$ Cancer 2011; 104:726-734.

5. Okugawa Y, Miki C, Toiyama Y, et al. Loss of tumoral expression of soluble IL-6 receptor is associated with disease progression in colorectal cancer. $\mathrm{Br}$ J Cancer 2010; 103:787-795.

6. Inoue Y, Miki C, Kusunoki M. Nutritional status and cytokine-related protein breakdown in elderly patients with gastrointestinal malignancies. J Surg Oncol 2004; 86:91-8.

7. Inoue $\mathrm{Y}$, Iwata T, Okugawa $\mathrm{Y}$, et al. Prognostic significance of a systemic inflammatory response in patients undergoing multimodality therapy for advanced colorectal cancer. Oncology 2013; 84:100-7. 